\title{
PD-1/PD-L1 Checkpoint in Breast Implant-Associated Anaplastic Large Cell Lymphoma: A Case Report
}

\author{
Antonella Bianchi ${ }^{1 *}$, Simona Ferrari ${ }^{1}$, Gabriella Gullotta ${ }^{1}$, Antonella Grasso ${ }^{2}$ and Ombretta Annibali ${ }^{3}$ \\ ${ }^{1}$ Unit of Pathology, University Hospital Campus Bio-Medico, Italy
}

${ }^{2}$ Breast Care Unit, University Hospital Campus Bio-Medico, Italy

${ }^{3}$ Unit of Hematology, Stem Cell Transplantation, University Hospital Campus Bio-Medico, Italy

Received: March 05, 2018; Published: March 16, 2018

*Corresponding author: Antonella Bianchi, Unit of Pathology, University Hospital Campus Bio-Medico, Via Alvaro del Portillo, 200, Rome, Italy, Tel: (+39) 0622541 1153; Email: a.bianchi@unicampus.it

\begin{abstract}
Breast implant-associated anaplastic large cell lymphoma (BI-ALCL) is a rare form of malignancy that arises around mammary implants in patients undergoing prosthetic surgery. It has been included as a provisional entity in the revised WHO classification of lymphoid malignancy. The PD-1/PD-L1 axis is one of the major mechanisms of immune escaping exerted by several cancer types in which up-regulation of PD-L1 is observed. We report the presence of PD-1/PD-L1 checkpoint in a case of BI-ALCL of a 67 year old woman, whom previously had undergone mastectomy and reconstruction for breast carcinoma.
\end{abstract}

Keywords: PD-1/PD-L1; BI-ALCL; Lymphomas; Lymphoprolipherative Diseases

Abbreviations: PD-1: Programmed Death-1; PD-L1: Programmed Death-Ligand 1; BI-ALCL: Breast Implant-Associated Anaplastic Large Cell Lymphoma; ALK: Anaplastic Lymphoma Kinase; TCR: T-Cell Receptor; CHL: Classical Hodgkin's Lymphoma; PTCL: Peripheral T-cell Lymphoma; FFPE: Fixed Formalin Paraffin Embedded

\section{Introduction}

Primary breast lymphomas are rare and account for approximately 0.04 to $0.5 \%$ of all breast cancers, with an estimated incidence of 1 case per 500.000 to 3 million women with implants [1]. The majority of these non-Hodgkin's lymphomas that affect the breast derive from B-lymphocytes and less than $10 \%$ are of T-cell origin [2]. In recent years, a distinct subset of T-cell lymphomas, so-called breast implant-associated anaplastic large cell lymphoma (BI-ALCL) has been described in women with breast implants. It was first described in 1997 and included in 2017 as a new provisional entity in the revised WHO classification of lymphoid malignancy within the group of the anaplastic large cell lymphoma (ALCL) [3]. ALCL is a specific subtype of peripheral T-cell lymphoma (PTCL) in which the different sub-entities, though sharing the presence of CD30-positive large atypical lymphocytes, substantially differ for clinical presentation (i.e. systemic, primary cutaneous, associated with breast implants) and behavior [4]. Among systemic ALCLs (sALCL), those harboring translocations of anaplastic lymphoma kinase gene (ALK) (ALK+ ALCL) have shown a more favourable clinical course as compared to those lacking ALK gene lesions (ALKALCL) [5].

BI-ALCL has an ALK-negative phenotype and, similarly to primary cutaneous ALCL (cALCL) [6], cases confined to the periimplant breast seroma fluid without invasion of the fibrous capsule have shown an excellent prognosis [7]. The PD-1/PD-L1 axis is one of the major mechanisms of immune escaping exerted by several cancer types in which up-regulation of PD-L1 is observed. Programmed death-1 (PD-1) receptors are expressed on activated $\mathrm{T}$ cells, and function to limit T-cell-mediated immune responses. PD-1 ligands, PD-L1 (B7-H1 or CD274) and PD-L2 (B7H3 or CD273), are physiologically expressed respectively by a subset of cells of the hematopoietic lineage (B cells, T cells and macrophages) and by antigen-presenting cells and epithelial tissues. They can be up-regulated in certain solid tumors and lymphomas. PD-L1 upon binding to PD-1 receptors, leads to the reduction of T-cell receptor (TCR) signaling, which is essential in maintaining immune tolerance and balanced responses to eliminate pathogens and tumor cells. 
There are Two Mechanisms of Expression of Immune Checkpoint Ligands on Tumors Cells

a) Constitutive expression of PD-L1 by tumors cells because of genetic alterations to the PD-L1 and PD-L2 locus of chromosome 9p24.1 (gains, amplifications or fusions);

b) Activation of signaling pathways, independent of inflammatory signals (intrinsic immune resistance) or through an induced response to inflammatory signals produced by an active anti-tumor immune response (adaptive immune resistance).

The disruption of PD-1/PD-L1 interaction using antibodies directed against PD-1 (Nivolumab and Pembrolizumab) or PDL1 (Atezolizumab) restores the $\mathrm{T}$ cell capability to exert cytolytic functions directed to neoplastic cells [8].

Furthermore, the assessment of PD-L1 expression through immunohistochemical staining is considered as the best biomarker that allows better selection of patients who are likely to respond to targeted immunotherapy, to improve treatment efficacy and to manage cost of therapies [9]. Although classical Hodgkin's lymphoma (cHL) is considered the prototype of lymphoid malignancies susceptible to blockade of PD-1/PD-L1 checkpoint [10] the success of checkpoint blockade therapy in the treatment of different solid tumors has encouraged the research for similar results in the wide spectrum of lymphoproliferative diseases [9,1113]. Our work provides the first evidence of the expression of PDL1 in BI-ALCL among the broad variety of lymphoid malignancies.

\section{Case Presentation}

The patient was a 67-year-old woman who noticed in 2010 a lump in the upper outer quadrant of her right breast. Clinical examination revealed a thick, movable $3 \times 3 \mathrm{~cm}$ nodule. Sonographic examination revealed a rounded hypoechoic lump $3 \mathrm{~cm}$ in diameter with irregular contour with increased perfusion; in the upper quadrants of the same breast it showed others two lesions with the same characteristics, $13.6 \mathrm{~mm}$ and $8 \mathrm{~mm}$ in diameter respectively, all suggesting a multicentric cancer. Mammograms confirm the presence of three lesions associated to microcalcifications. The subsequent cytology reported the diagnosis of carcinoma. The patient underwent mastectomy and axillary dissection plus reconstruction by tissue expander. Histopathology reported multiple high grade ductal infiltrating carcinoma associated to high grade ductal carcinoma in situ and microscopic aspects of lymphovascular invasion; on immunohistochemistry, tumor cells expressed estrogen and progesterone receptors, Ki67 15\%, HER2 negative (Luminal A-like subtype). All 16 lymph nodes removed were negative. She received ormonotherapy with an Aromatase inhibitor until February 2015.

In 2011 the patient underwent the second stage reconstruction with silicone implant placement and contra lateral symmetrisation. The follow up was negative until February 2017 when two residual auxiliary lymph node were revealed by ultrasound examination associated with the presence of peri-prosthetic effusion, found in a control CT scan. Whole body positron emission tomographycomputed tomography (PET/CT) scan with Huorodeoxyglucose (FDG) radiotracer revealed increased FDG uptake only in the axillary region with a maximum SUV of 6.4 and in the external quadrants of the right breast.

For this reason, the patient underwent first diagnostic dissection of palpable lymph nodes and then to lymphadenectomy and capsulectomy (Figure 1) with extraction of silicone implant and drainage of peri-prosthetic fluid. Histopathologically, clusters of lymphoma cells were present in the peri-prosthetic fluid and in the fibrous capsule surrounding the implant, but not beyond it. The lymph node was characterized by intrasinusal infiltrate of highly pleomorphic cells with horseshoe-shaped and similReed-Sternberg nuclei, with prominent nucleoli. The tumour cells were immunohistochemically positive for CD30, CD15, CD4, CD5, Perforin, Granzyme, MUM1/IRF4 and demonstrated negative immunostaining for ALK, LMP1, pan-B-cell and epithelial markers. Others T-cell markers were down regulated.

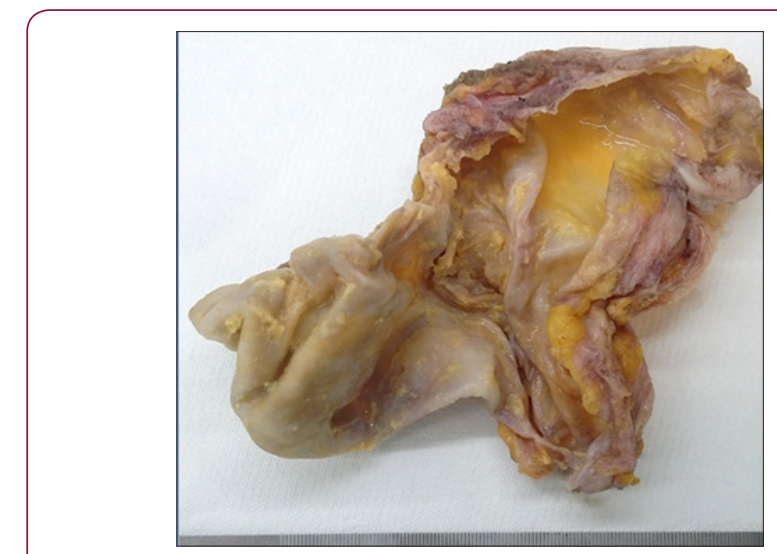

Figure 1: The excised BI-ALCL capsular tissue.

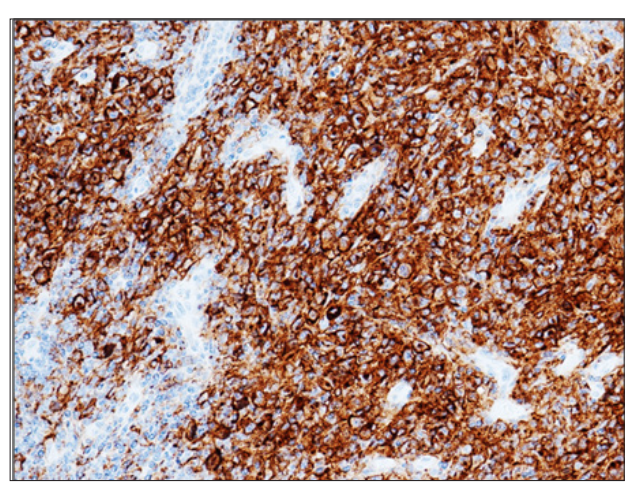

Figure 2: The neoplastic cells exhibiting strong membranous staining immunoreactivity for PD-L1 (22C3 DAKO). (200 X).

Almost all tumor cells $( \pm 90 \%)$ showed expression for PDL1 [22C3 DAKO, Agilent Technologies] with specific, strong, and fully membranous staining pattern (Figure 2) [11,13]. PD-L1 expression was also evaluated in non-neoplastic cells. A weak membranous partial stain was observed in $\pm 20 \%$ of adjacent nodal histiocytes [14]. Immunohistochemistry was performed with the automated device Dako Omnis (Agilent Technologies') 
to ensure repeatability and reproducibility. Appropriate positive and negative controls were routinely included for intra-laboratory optimization of the tests. The staging at the time of diagnosis, according to Clemens et al., was T3, N1, M0 [7]. Bone marrow biopsy showed no evidence of lymphoma involvement. As patients with positive regional lymphnode involvement at diagnosis have a higher rate of recurrence, CHOP systemic treatment has been the therapeutic option chosen after capsulectomy. The patient, still under treatment when publishing, will continue close surveillance following completion of chemotherapy.

\section{Discussion}

BI-ALCL is considered a disease of the fibrous capsule surrounding the implant and not of the breast parenchyma. Silicone might be immunogenic stimulating a chronic inflammation and, such as Helicobacter pylori infection in gastric extra nodal marginal zone lymphoma, could be associated with development of lymphomas Moreover, ALCL originates from activated mature cytotoxic T cells [15]. The overexpression of PD-L1 has never been described before in BI-ALCL between the wide spectrums of lymphoproliferative diseases. It could be related to the deregulation of genes involved in the inflammatory reaction, well known in BI-ALCL, supporting an immune pathogenetic mechanism of lymphomagenesis [16].

Besides these intriguing aspects, since targeted therapy has recently shown encouraging results, as, for example, BrentuximabVedotin (BV) for cases overexpressing CD30 antigen, also PD-L1 assessment by immunohistochemistry could be proposed as a predictive assay for selecting patients who do not have responded to conventional chemotherapy, potentially treatable with checkpoint blockade therapies [17]. Due to this essential role as biomarker, from a technical point of view, all pre-analytical (tissue fixation, handling, processing and treatment of histological sections) and analytical issues (immunohistochemical visualization system, commercial staining platform, chromogen, primary antibody, pre-treatment) must be carefully considered in the pathology laboratory when immunohistochemical tests are performed on FFPE (fixed formalin paraffin embedded) tissue. Moreover, morphologic evaluation is fundamental to correctly apply a scoring algorithm in an exhaustive pathologic report.

\section{Conclusion}

In conclusion, however further studies would be necessary for the validation of reporting criteria, pathologists and haematologists should continue to collaborate for the optimization of the PD-L1 immunohistochemical assays for the patients affected by different subtypes of lymphomas and considered for immunotherapy.

\section{Acknowledgement}

The authors are deeply grateful to Alessandra Innocenzi for excellent technical assistance.

\section{References}

1. Brody GS (2015) Anaplastic large cell lymphoma occurring in women with breast implants: analysis of 173 cases. Plast Reconstr Surg 135(3): 695-705.

2. Evren S (2017) Breast Implant-Associated Anaplastic Large Cell Lymphoma (ALCL): A Case Report. Am J Case Rep 18: 605-610.

3. Swerdlow SH, Campo E, Harris NL (2017) WHO Classification of Tumours of Haematopoietic and Lymphoid Tissues. (Eds.), World Health Organization Classification of Tumours, Revised ( $4^{\text {th }}$ Edn). Lyon, France.

4. Miranda RN (2014) Breast implant-associated anaplastic large-cell lymphoma: long-term follow-up of 60 patients. J Clin Oncol 32(2): 114-120.

5. Morris SW (1994) Fusion of a kinase gene, ALK, to a nucleolar protein gene, NPM, in non Hodgkin's lymphoma. Science 263(5151): 1281-1284.

6. Savage KJ (2008) ALK- anaplastic large-cell lymphoma is clinically and immunophenotypically different from both ALK+ ALCL and peripheral T-cell lymphoma, not otherwise specified: report from the International Peripheral T-Cell Lymphoma Project. Blood 111(12): 5496-5504.

7. Clemens MW (2016) Complete Surgical Excision Is Essential for the Management of Patients With Breast Implant-Associated Anaplastic Large-Cell Lymphoma. J Clin Oncol 34(2): 160-168.

8. Bianchi A (2017) PD-1/PD-L1 checkpoint in lymphoproliferative malignancies: focus on critical points for tissue assessment. ARC Journal of Haematology 2(2): 1-6.

9. Gravelle P (2017) Mechanisms of PD-1/PD-L1 expression and prognostic relevance in non-Hodgkin lymphoma: a summary of immunohistochemical studies. Oncotarget 8(27): 44960-44975.

10. Ansell SM (2015) PD-1 blockade with nivolumab in relapsed or refractory Hodgkin's lymphoma. N Engl J Med 372(4): 311-319.

11. Menter T (2016) Evaluation of the diagnostic and prognostic value of PDL1 expression in Hodgkin and B-cell lymphomas. Hum Pathol 54: $17-24$

12. Bledsoe JR (2016) The immunophenotypic spectrum of primary mediastinal large B-cell lymphoma reveals prognostic biomarkers associated with outcome. Am J Hematol 91(10): E436-E441.

13. Vranic S (2016) PD-L1 Status in Refractory Lymphomas. PLos One 11(11): e0166266.

14. Kim WY (2016) Expression of programmed cell death ligand 1 (PD-L1) in advanced stage EBV-associated extranodal NK/T cell lymphoma is associated with better prognosis. Virchows Arch 469(5): 581-590.

15. Xu J, S Wei (2014) Breast implant-associated anaplastic large cell lymphoma: review of a distinct clinicopathologic entity. Arch Pathol Lab Med 138(6): 842-846.

16. Di Napoli A (2018) Targeted next generation sequencing of breast implant-associated anaplastic large cell lymphoma reveals mutations in JAK/STAT signalling pathway genes, TP53 and DNMT3A. Br J Haematol 180(5): 741-744.

17. Ezekwudo DE (2017) Breast Implant-Associated Anaplastic Large Cell Lymphoma: A Case Report and Review of the Literature. Case Rep Oncol Med 2017: 6478467. 


\section{(c) (i) This work is licensed under Creative}

Submission Link: https://biomedres.us/submit-manuscript.php

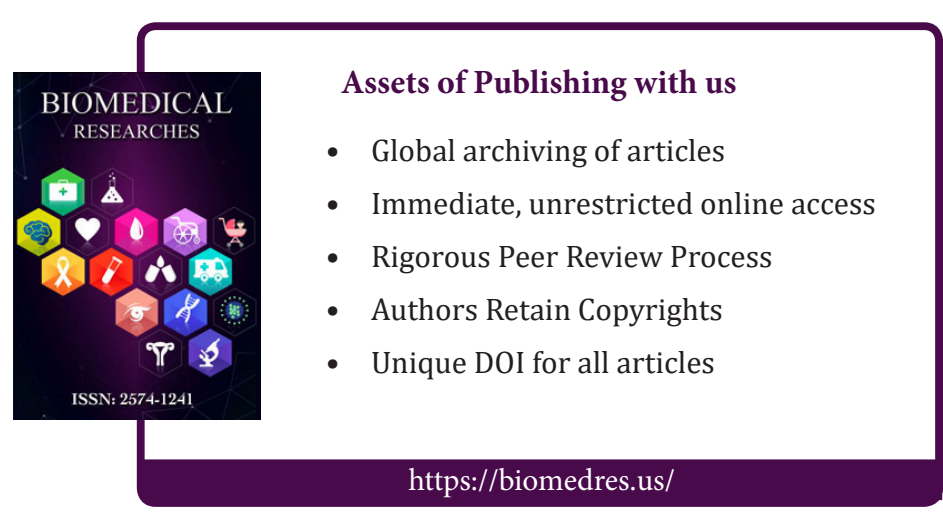

